\title{
Homology exponents for $H$-spaces
}

\section{Alain Clément and Jérôme Scherer}

\begin{abstract}
We say that a space $X$ admits a homology exponent if there exists an exponent for the torsion subgroup of $H^{*}(X ; \mathbb{Z})$. Our main result states that if an $H$-space of finite type admits a homology exponent, then either it is, up to 2-completion, a product of spaces of the form $B \mathbb{Z} / 2^{r}, S^{1}, \mathbb{C} P^{\infty}$, and $K(\mathbb{Z}, 3)$, or it has infinitely many non-trivial homotopy groups and $k$-invariants. Relying on recent advances in the theory of $H$-spaces, we then show that simply connected $H$-spaces whose mod 2 cohomology is finitely generated as an algebra over the Steenrod algebra do not have homology exponents, except products of mod 2 finite $H$-spaces with copies of $\mathbb{C} P^{\infty}$ and $K(\mathbb{Z}, 3)$.
\end{abstract}

\section{Introduction}

The study of the torsion in the homotopy groups and the integral homology groups of a space motivated the Moore conjecture, see [26], and the Serre conjecture, [27]. Serre proved that a simply connected space with finite dimensional (and non-trivial) mod $p(\mathrm{co})$ homology $H^{*}\left(X ; \mathbb{F}_{p}\right)$ must have infinitely many non-trivial homotopy groups. He conjectured that there should in fact exist infinitely many homotopy groups of $X$ containing $p$-torsion, which was proved eventually by McGibbon and Neisendorfer [24], relying on Miller's solution [22] of the Sullivan conjecture. This was then refined further by Lannes and Schwartz in [21]. Their criterion is that $H^{*}\left(X ; \mathbb{F}_{p}\right)$ is locally finite, as a module over the Steenrod algebra. Dwyer and Wilkerson went one step further, [9], looking only at the module $Q H^{*}\left(X ; \mathbb{F}_{p}\right)$ of indecomposable elements. Félix, Halperin, Lemaire, and Thomas provided yet another criterion involving the depth of $H_{*}\left(\Omega X ; \mathbb{F}_{p}\right),[12]$. In their subsequent paper [13] they focused on the size of the torsion part in the "loop

2000 Mathematics Subject Classification: Primary 57T25, 55S45; Secondary 55P20, 55S10, 55T10, 55T20.

Keywords: Homology exponent, $H$-space, loop space, Steenrod algebra. 
space homology" $H_{*}(\Omega X ; \mathbb{Z})$. They proved in fact a homological version of the Moore conjecture, namely that the $p$-torsion part of the integral homology of the loop space of a $\mathbb{Z}_{(p)}$-elliptic space always has an exponent.

In this article we are interested in understanding when the torsion subgroup of the integral homology of a large class of loop spaces, and more generally $H$-spaces, can have an exponent. In the spirit of Serre's theorem, we first classify those $H$-spaces having a homology exponent at the prime 2 which are Postnikov pieces (they have only a finite number of non-trivial homotopy groups). Thus we will say henceforth that a space admits a homology exponent if there exists an integer $k$ such that $2^{k} \cdot T_{2} H^{*}(X ; \mathbb{Z})=0$, where $T_{2}$ stands for the 2-torsion subgroup. We work with connected $H$-spaces of finite type.

Theorem 5.2 Let $X$ be an $H$-space of finite type which admits a homology exponent. Then either $X$ is, up to 2-completion, a product of spaces of the form $B \mathbb{Z} / 2^{r}, S^{1}, \mathbb{C} P^{\infty}$ and $K(\mathbb{Z}, 3)$, or $X$ admits infinitely many non-trivial $k$-invariants and homotopy groups.

The methods we develop predict in fact explicit degrees in which to find homology classes of order $2^{r}$ for arbitrarily large $r$ when the space has no homology exponent, quite in the spirit of Browder's "infinite implications", [3]. This builds on previous work by the first author, who analyzed the case of a Postnikov piece with at most two non-trivial homotopy groups in [8].

There is a class of $H$-spaces which is very close to the Postnikov pieces we have been dealing with up to now, namely those $H$-spaces for which the mod 2 cohomology is finitely generated as an algebra over the Steenrod algebra. They are obtained indeed as extensions by $H$-fibrations of an $H$ space with finite mod 2 cohomology by a Postnikov piece, [5].

Theorem 7.5 Let $X$ be a simply connected $H$-space of finite type such that $H^{*}\left(X ; \mathbb{F}_{2}\right)$ is finitely generated as an algebra over the Steenrod algebra. Assume that $X$ admits a homology exponent. Then $X$ is, up to 2-completion, the product of a mod 2 finite $H$-space $Y$ with copies of $K(\mathbb{Z}, 2)$ and $K(\mathbb{Z}, 3)$.

This contrasts with the homological version of the Moore conjecture obtained by Félix, Halperin, and Thomas in [13]. Of course the mod 2 cohomology of the loop space on a finite complex is very rarely finitely generated as an algebra over the Steenrod algebra. In this article we only deal with the prime 2, just like Serre in [27], or Lannes and Schwartz in [21]. We expect our methods to go through mutatis mutandis in the case of an odd prime.

Acknowledgements. We would like to thank Richard Kane for providing a simple proof of Lemma 3.3, and Juan A. Crespo and Wolfgang Pitsch for helpful comments. The second author would like to thank Kathryn Hess and the IGAT, EPFL, for the invitation which made this collaboration possible. 


\section{Reduction to simply connected spaces}

In this section we explain how to reduce the study of arbitrary connected $H$-spaces to simply connected ones for which the second homotopy group is torsion. These are then the spaces we study in the rest of the article. Let us start with basic terminology and notation.

Notation 1.1. A space $X$ is a Postnikov piece if it has only finitely many non-trivial homotopy groups. It is an $H$-Postnikov piece if it is moreover an $H$-space. The $n$-th Postnikov section $i_{n}: X \rightarrow X[n]$ is determined, up to homotopy, by the property that it induces isomorphisms on homotopy groups $\pi_{i}$, for $i \leq n$, and $\pi_{i} X[n]=0$ for $i>n$. The homotopy fiber $X\langle n\rangle$ of $i_{n}$ is the $n$-connected cover of $X$. When $X$ is simple (for example when $X$ is simply connected or when $X$ is an $H$-space), there exist $k$-invariants $k_{n} \in H^{n+1}\left(X[n-1] ; \pi_{n} X\right)$ such that $X[n]$ can be recovered as the homotopy fiber of a map $k_{n}: X[n-1] \rightarrow K\left(\pi_{n} X, n+1\right)$ representing the $k$-invariant. When $X$ is an $H$-space, all $k$-invariants are primitive elements.

Let $X$ be a space. By $\left\{B_{r}^{*}, d_{r}\right\}$ we denote its mod 2 cohomology Bockstein spectral sequence: $B_{1}^{*} \cong H^{*}\left(X ; \mathbb{F}_{2}\right) \Longrightarrow\left(H^{*}(X ; \mathbb{Z}) /\right.$ torsion $) \otimes \mathbb{F}_{2}$. Recall that the first differential $d_{1}=S q^{1}$ is the Bockstein and a pair of elements $x$ and $y$ which survive to the page $B_{r}$ and such that $d_{r}(x)=y$ detect a copy of $\mathbb{Z} / 2^{r}$ in $H^{*}(X ; \mathbb{Z})$ in degree $|y|=|x|+1$.

We collect now a result about "small" Postnikov pieces. These will turn out to be the only $H$-Postnikov pieces having an exponent.

Proposition 1.2. Let $X$ be a connected $H$-space of finite type such that $\pi_{2}(X)$ and $\pi_{3}(X)$ are torsion free. Then $X[3]$ is a product of spaces of the form $B \mathbb{Z} / p^{r}, S^{1}, \mathbb{C} P^{\infty}$ and $K(\mathbb{Z}, 3)$. Moreover, $X[3]$ admits a homology exponent.

Proof. It is well-known that a copy of the integers in $\pi_{1} X$ corresponds to a copy of $S^{1}$ splitting of $X$ (because of the existence of a section $S^{1} \rightarrow X$ ). One readily verifies that $H^{3}\left(K\left(\mathbb{Z} / p^{r}, 1\right) ; \mathbb{Z}\right)=0$, which shows that the first $k$ invariant must be trivial. Thus $X[2]$ splits as a product of copies of $B \mathbb{Z} / p^{r}$ 's, $S^{1}$ 's, and $\mathbb{C} P^{\infty}$ 's. Next, the only elements in $H^{4}\left(K\left(\mathbb{Z} / p^{r}, 1\right) ; \mathbb{Z}\right)$, for any prime $p$ and any integer $r$, and $H^{4}(K(\mathbb{Z}, 1) ; \mathbb{Z})$ are multiples of the square of the generator in degree 2 . Such elements are not primitive (unless they are trivial), and hence cannot be the $k$-invariants of an $H$-space. Therefore, the second $k$-invariant of $X[3]$ is trivial as well and the space splits as a product.

It remains to prove the assertion about the homology exponent. Recall that $p^{r} \cdot \widetilde{H}^{*}\left(B \mathbb{Z} / p^{r} ; \mathbb{Z}\right)=0$ (by a transfer argument $), H^{*}\left(S^{1} ; \mathbb{Z}\right), H^{*}\left(\mathbb{C} P^{\infty} ; \mathbb{Z}\right)$ are torsion free and $2 \cdot T_{2}\left(H^{*}(K(\mathbb{Z}, 3) ; \mathbb{Z})\right)=0$ (as a consequence of Serre $[27]$ or Cartan's computations [4]). 
Lemma 1.3. Let $r \geq 1$ and $\left(S^{1}\right)^{r} \rightarrow Y \rightarrow X$ be a $H$-fibration. If $X$ admits a homology exponent, then so will $Y$.

Proof. Let us remark that the fibration is orientable [29, p. 476]. We obtain the result for $r=1$ by inspecting the associated Gysin cohomology exact sequence and conclude by induction on $r$.

We conclude this section with the promised reduction. The existence of a homology exponent for an arbitrary $H$-space is detected in the homology of a certain covering space.

Proposition 1.4. Let $X$ be a connected H-space of finite type. Then there exists a simply connected $H$-space of finite type $Y$ such that $\pi_{2} Y$ is a torsion abelian group and $Y$ fits into the following $H$-fibration:

$$
Y \longrightarrow X \longrightarrow B \pi_{1} X \times K\left(\mathbb{Z}^{k}, 2\right),
$$

for some $k \geq 0$. Moreover, if $X$ admits a homology exponent, then so will $Y$.

Proof. Let us first deal with the copies of $\mathbb{Z}$ in $\pi_{1} X$. They correspond to a torus $\left(S^{1}\right)^{r}$ splitting off $X$. There exists hence $X_{1}$ such that $X \simeq X_{1} \times\left(S^{1}\right)^{r}$ and $\pi_{1} X_{1} \cong \oplus_{a} \mathbb{Z} / 2^{s_{a}} \oplus A$, where $A$ is a $2^{\prime}$-torsion abelian group (i.e. any element in $A$ has finite order prime to 2). There is a map $B \mathbb{Z} / 2^{s_{a}} \rightarrow K(\mathbb{Z}, 2)$ for any $a$, corresponding to the Bockstein operation of order $s_{a}$. Let us define $X_{2}$ to be the homotopy fiber of the composite map

$$
X_{1} \rightarrow B \pi_{1} X_{1} \rightarrow \prod_{a} K(\mathbb{Z}, 2) \times K(A, 1) .
$$

As in the proof of [21, Proposition 0.7], $X_{2}$ splits as a product $\widetilde{X} \times \prod_{a} S^{1}$, where $\widetilde{X}$ denotes the universal cover of $X$. By the previous lemma, $X_{2}$, and thus $\tilde{X}$, admit an exponent if $X$ does.

Finally write $\pi_{2} X \cong \mathbb{Z}^{k} \oplus A^{\prime}$ where $A^{\prime}$ is a finite torsion group and let $Y$ be the homotopy fiber of the map $\widetilde{X} \rightarrow K\left(\mathbb{Z}^{k}, 2\right)$ so that $\pi_{2} Y \cong A^{\prime}$. The previous lemma yields the statement about the exponent. The description of the base of the $H$-fibration comes from Proposition 1.2.

\section{A splitting principle}

Let $X$ be a Postnikov piece, which highest non-trivial homotopy group is $\pi_{n} X=H$. In this section we show that, if $H$ is torsion free, the $(n-2)$ connected cover $X\langle n-2\rangle$ splits as a product $K(H, n) \times K(G, n-1)$. Loosely speaking, the $n$-th $k$-invariant attaches $K(H, n)$ directly to $X[n-2]$ and cannot tie the last two homotopy groups together. 
We will first need some basic results on Eilenberg-Mac Lane spaces. We follow the terminology and notation of [25, Chapter 1]. For a unified treatment of the spaces $K\left(\mathbb{Z} / 2^{s}, n\right)$, with $s \geq 1$, and $K(\mathbb{Z}, n)$, it is convenient to introduce a notation for the higher Bockstein operations. Let $u_{n}$ (respectively $S q_{s}^{1} u_{n}$ ) denote the generator of the 1-dimensional $\mathbb{F}_{2}$-vector space $H^{n}\left(K\left(\mathbb{Z} / 2^{s}, n\right) ; \mathbb{F}_{2}\right)$ (respectively $H^{n+1}\left(K\left(\mathbb{Z} / 2^{s}, n\right) ; \mathbb{F}_{2}\right)$ ). For an admissible sequence $I=\left(i_{1}, \ldots, i_{m}\right)$, we will write $S q_{s}^{I} u_{n}$ instead of $S q^{\left(i_{1}, \ldots, i_{m-1}\right)} S q_{s}^{1} u_{n}$ if $i_{m}=1$ and instead of $S q^{I} u_{n}$ if $i_{m} \neq 1$. We denote also by $u_{n}$ the generator of $H^{n}\left(K(\mathbb{Z}, n) ; \mathbb{F}_{2}\right)$.

Serre computed the mod 2 cohomology of Eilenberg-Mac Lane spaces.

Theorem 2.1. (Serre, [27]) Let $n \geq 1$ and $s \geq 1$.

(1) The $\mathbb{F}_{2}$-algebra $H^{*}\left(K\left(\mathbb{Z} / 2^{s}, n\right) ; \mathbb{F}_{2}\right)$ is isomorphic to the polynomial $\mathbb{F}_{2}$-algebra on generators $S q_{s}^{I} u_{n}$, where $I$ covers all the admissible sequences of excess $e(I)<n$.

(2) The $\mathbb{F}_{2}$-algebra $H^{*}\left(K(\mathbb{Z}, n) ; \mathbb{F}_{2}\right)$ is isomorphic to the polynomial $\mathbb{F}_{2^{-}}$ algebra on generators $S q^{I} u_{n}$, where $I$ covers all the admissible sequences of the form $\left(i_{1}, \ldots, i_{n}\right)$ where $i_{n} \neq 1$ and of excess $e(I)<n$.

Our first lemma relies on Serre's computations in low degrees.

Lemma 2.2. Let $G$ be a finitely generated abelian group, $H$ be free abelian, and $n \geq 3$. Then $P^{n+1} H^{*}(K(G, n-1) ; H)=0$.

Proof. Since $H^{*}(K(G, n-1) ; H) \cong H^{*}(K(G, n-1) ; \mathbb{Z}) \otimes H$, it is enough to consider the case when $H=\mathbb{Z}$. When $n \geq 4$, the only elements in $H^{n+1}\left(K(G, n-1) ; \mathbb{F}_{2}\right)$ are sums of elements of the form $S q^{2} u_{n-1}$. These elements all have non-trivial Bockstein and we see therefore from the Bockstein spectral sequence that $H^{n+1}(K(G, n-1) ; \mathbb{Z})$ is 2 -torsion free. As there is obviously no odd primary torsion in this degree (from Cartan's description of $\left.H^{*}\left(K(G, n-1) ; \mathbb{F}_{p}\right),[4]\right)$, we have that $H^{n+1}(K(G, n-1) ; \mathbb{Z})=0$. When $n=3$, write $G \cong \mathbb{Z}^{k} \oplus A$, where $A$ is torsion. Then $H^{4}(K(G, 2) ; \mathbb{Z}) \cong$ $H^{4}\left(K\left(\mathbb{Z}^{k}, 2\right) ; \mathbb{Z}\right)$. There are no primitive elements in this degree.

Proposition 2.3. Let $X$ be a simply connected $H$-space of finite type such that $\pi_{2} X$ is a torsion group. Let $n \geq 3$, consider the Postnikov section $X[n]$ and assume that $\pi_{n} X$ is torsion free. Then we have the following $H$-fibration:

$$
X[n] \longrightarrow X[n-2] \longrightarrow K\left(\pi_{n-1} X, n\right) \times K\left(\pi_{n} X, n+1\right) .
$$


Proof. Set $G=\pi_{n-1} X$ and $H=\pi_{n} X$. We prove that the $k$-invariant $k_{n} \in H^{n+1}(X[n-1] ; H)$ factors through $H^{n+1}(X[n-2] ; H)$. Let us consider the fibration

$$
K(G, n-1) \rightarrow X[n-1] \rightarrow X[n-2]
$$

and the cofibration $K(G, n-1) \rightarrow X[n-1] \rightarrow C$. From Lemma 2.2 we deduce that the $H$-map $K(G, n-1) \rightarrow X[n-1] \stackrel{k_{n}}{\longrightarrow} K(H, n+1)$ is null-homotopic.

Therefore $k_{n}$ factors through a map $C \rightarrow K(H, n+1)$. By Ganea's result [14] the fiber of the map $C \rightarrow X[n-2]$ is the join $K(G, n-1) *$ $\Omega(X[n-2])$. This is an $n$-connected space and because $\pi_{2} X$ is a torsion group so is $\pi_{n+1}(K(G, n-1) * \Omega(X[n-2])) \cong G \otimes \pi_{2} X$. Thus $K(H, n+1)$ is a $K(G, n-1) * \Omega(X[n-2])$-local space, as we assume that $H$ is torsion free. From Dwyer's version of Zabrodsky lemma [10, Proposition 3.5] we deduce that $k_{n}$ factors through a map $k: X[n-2] \rightarrow K(H, n+1)$.

If $k_{n-1} \in H^{n}(X[n-2] ; G)$ denotes the previous $k$-invariant, this means that $X[n]$ is the homotopy fiber of $X[n-2] \stackrel{k_{n-1} \times k}{\longrightarrow} K(G, n) \times K(H, n+1)$, the product map.

\section{Gaps in the primitives}

This section contains the key cohomological result which makes the analysis of the Serre spectral sequence possible. We notice first that there are gaps in the mod 2 cohomology of Eilenberg-Mac Lane spaces and show then that these gaps propagate in the cohomology of any Postnikov piece.

Definition 3.1. Let $n \geq 1$. We set $A_{n}=\left\{a \in \mathbb{N}, a\right.$ odd $\left.\mid \nu_{2}(a) \geq n+1\right\}$ where $\nu_{2}(a)$ is the 2-adic length of the integer $a$.

We will show that there are no indecomposable elements in the cohomology of an $n$-stage Postnikov piece in degrees $a \in A_{n}$. To deduce that there are no primitive elements either, we make use of the relationship provided by the Milnor-Moore theorem [23, Proposition 4.21]: For a connected, associative, and commutative Hopf algebra over $\mathbb{F}_{2}$, there is an exact sequence of graded modules

$$
0 \longrightarrow P(\xi H) \longrightarrow P H \longrightarrow Q H,
$$

where $\xi H$ is the image of the Frobenius map $\xi: x \mapsto x^{2}, Q H$ is the module of indecomposable elements and $P H$ is the module of primitive elements of $H$.

Lemma 3.2. Let $H$ be a finitely generated abelian group and $n \geq 2$. Then

$$
Q^{a} H^{*}\left(K(H, n) ; \mathbb{F}_{2}\right)=0=P^{a} H^{*}\left(K(H, n) ; \mathbb{F}_{2}\right)
$$

for all $a \in A_{n}$. 
Proof. When it is not trivial, the $\mathbb{F}_{2}$-algebra structure of $H^{*}\left(K(H, n) ; \mathbb{F}_{2}\right)$ is given by a polynomial algebra on generators of the form $S q_{s}^{I} u_{n}$ where $I$ runs over admissible sequences with excess $e(I)<n$, as we have recalled in Theorem 2.1. Careful calculations show that these generators lie in degrees $1+2^{h_{1}}+\cdots+2^{h_{n-1}}$ where $h_{1} \geq \cdots \geq h_{n-1} \geq 0$ (see [27, Théorème $1, \mathrm{p} .212$ and Théorème 2, p.213]). The 2-adic length of these degrees is bounded by $n$. This shows that there are no indecomposable elements in the degrees we claimed. These degrees being odd, there are no primitives either, because the kernel of the map $P H^{*}\left(K(H, n) ; \mathbb{F}_{2}\right) \rightarrow Q H^{*}\left(K(H, n) ; \mathbb{F}_{2}\right)$ is concentrated in even degrees.

The proof of the following lemma has been kindly communicated to us by Richard Kane, [18].

Lemma 3.3. Let $B$ be a connected, associative, and commutative Hopf algebra of finite type over $\mathbb{F}_{2}$ and $A$ a sub-Hopf algebra of $B$. Then the morphism $Q A \rightarrow Q B$ is injective in odd degrees.

Proof. We work in degree $2 n+1$. Consider the Hopf subalgebra $C$ of $A$, and hence of $B$, generated by the elements in $A$ of degree $\leq 2 n$. Then one has an inclusion of quotient Hopf algebras $A / / C \hookrightarrow B / / C$ by [17, Corollary p.9]. Let $x$ be an indecomposable element in $Q A$ of degree $2 n+1$. It determines a non-zero primitive element in $P(A / / C)$, hence in $P(B / / C)$. As the map $P(B / / C) \rightarrow Q(B / / C)$ is injective in odd degrees, we see that the composite $Q A \rightarrow Q(B / / C)$ is injective in degree $2 n+1$. Therefore $Q A \rightarrow Q B$ must be injective in degree $2 n+1$ as well.

Remark 3.4. The preceding lemma has a nice interpretation in terms of André-Quillen homology, the derived functor of $Q(-)$. It is proved in [6, Proposition 1.3] that one has, in the setting of the lemma, an exact sequence $H_{1}^{Q}(B / / A) \rightarrow Q A \rightarrow Q B \rightarrow Q(B / / A) \rightarrow 0$, a result dual to that of Bousfield, [1, Theorem 3.6]. Moreover the graded $\mathbb{F}_{2}$-vector space $H_{1}^{Q}(B / / A)$ is concentrated in even degrees.

We are now ready to prove that the gaps also appear in the cohomology of any Postnikov piece. A good example to have in mind, in fact for the more general situation of Proposition 7.2, is that of the Postnikov fibration $S^{3}\langle 3\rangle \rightarrow S^{3} \rightarrow K(\mathbb{Z}, 3)$. Recall that $H^{*}\left(S^{3}\langle 3\rangle ; \mathbb{F}_{2}\right) \cong \mathbb{F}\left[x_{4}\right] \otimes E\left(x_{5}\right)$. In the notation of the following proof, the polynomial algebra $\mathbb{F}\left[x_{4}\right]=S$ and the exterior algebra $E\left(x_{5}\right)=\Lambda$.

Proposition 3.5. Let $n \geq 2$ and $X$ be a simply connected n-stage $H$ Postnikov piece of finite type. Then $Q^{a} H^{*}\left(X ; \mathbb{F}_{2}\right)=0=P^{a} H^{*}\left(X ; \mathbb{F}_{2}\right)$ for all $a \in A_{n}$. 
Proof. The proof goes by induction on $n$. We have the following $H$-fibration given by the Postnikov tower of $X$ :

$$
X \stackrel{p}{\longrightarrow} X[n-1] \stackrel{k}{\longrightarrow} K\left(\pi_{n}(X), n+1\right) .
$$

We rely on the analysis of the Eilenberg-Moore spectral sequence done by Smith [28, Proposition 3.2]. One can identify the quotient Hopf algebra $R=H^{*}\left(X[n-1] ; \mathbb{F}_{2}\right) / / \operatorname{ker} p^{*}$ via $p^{*}$ with a sub-Hopf algebra of $H^{*}\left(X ; \mathbb{F}_{2}\right)$. The corresponding quotient $H^{*}\left(X ; \mathbb{F}_{2}\right) / / R$ splits, as an algebra, as a tensor product $\Lambda \otimes S$, where $S$ is a sub-Hopf algebra (and a sub- $\mathcal{A}_{2}$-algebra) of $H=H^{*}\left(K\left(\pi_{n}(X), n\right) ; \mathbb{F}_{2}\right)$ and $\Lambda$ is an exterior algebra. Let $K$ denote the (polynomial) Hopf algebra kernel of $k^{*}$. Its generators which do not lie in the kernel of the cohomology suspension $H^{*}\left(K\left(\pi_{n}(X), n+1\right) ; \mathbb{F}_{2}\right) \rightarrow H^{*-1}\left(K\left(\pi_{n}(X), n\right) ; \mathbb{F}_{2}\right)$-such as the generators of maximal excess- correspond to the generators of the polynomial algebra $S$, and the ones in the kernel correspond to generators of $\Lambda$, with a shift by one of the degree.

Since $Q(-)$ is right exact, we only have to prove that $Q R, Q S$, and $Q \Lambda$ are trivial in degrees in $A_{n}$. First, $Q^{a} H^{*}\left(X[n-1] ; \mathbb{F}_{2}\right)=0$ for any $a \in A_{n-1}$ by induction hypothesis and $Q H^{*}\left(X[n-1] ; \mathbb{F}_{2}\right) \rightarrow Q R$ is a surjection. We conclude that $Q^{a} R=0$ for any $a \in A_{n}$ since $A_{n} \subset A_{n-1}$. Second, we apply the preceding lemma to the inclusion $S \subset H$ and see that $Q S \rightarrow Q H$ is a monomorphism in odd degrees. Therefore $Q^{a} S=0$ for all $a \in A_{n}$ by Lemma 3.2. Finally we examine the generators of the exterior algebra $\Lambda$. Because the only relations in a Hopf algebra are of truncated polynomial type, one sees that the degrees in which such generators lie are of the form $2^{n}$ times the degree of some generator of non-maximal excess in $H^{*}\left(K\left(\pi_{n}(X), n+1\right)\right.$. The same computation as in the proof of Lemma 3.2 shows then that the 2 -adic length of such an element is smaller than $n+1$.

\section{Transverse elements in Eilenberg-Mac Lane spaces}

Now begins the study of the 2-torsion in Postnikov pieces. In this section we deal with the first step of the induction, namely the analysis of the case of Eilenberg-Mac Lane spaces. Recall that $\left\{B_{r}^{*}, d_{r}\right\}$ denote the mod 2 cohomology Bockstein spectral sequence of a space $X$.

Definition 4.1. Let $n$ and $r$ be two positive integers. An element $x \in B_{r}^{n}$ is said to be $\ell$-transverse if $d_{r+l} x^{2^{l}} \neq 0 \in B_{r+l}^{2^{l} n}$ for all $0 \leq l \leq \ell$. An element $x \in B_{r}^{n}$ is said to be transverse if it is $\ell$-transverse for all $\ell \geq 0$. We will also speak of transverse implications of an element $x \in B_{r}^{n}$. 
Every transverse element gives rise to 2-torsion of arbitrarily high order in the integral cohomology of $X$. This definition, introduced in [7], adapts Browder's "infinite implications" from [3] to our purpose. To us, the fact that the elements die in increasing pages of the Bockstein spectral sequence is crucial, whereas Browder was merely interested to know that the degrees of the elements was increasing.

Our strategy for disproving the existence of a homology exponent for a space will consist in exhibiting a transverse element in its mod 2 cohomology Bockstein spectral sequence. Note that in principle the absence of transverse elements does not imply the existence of an exponent for the 2-torsion part in $H^{*}(X ; \mathbb{Z})$. An easy example if given by the infinite wedge $\vee M\left(\mathbb{Z} / 2^{n}, n\right)$.

In the special case of Eilenberg-Mac Lane spaces, we have the following result, which follows from Cartan's results [4] on the integral homology of Eilenberg-Mac Lane spaces and careful bookkeeping in the Bockstein spectral sequence.

Theorem 4.2. (Clément, [7, Theorem 1.3.2]) Let $H$ be an abelian group of finite type, whose 2 -torsion part is thus of the form $\mathbb{Z} / 2^{s_{1}} \oplus \cdots \oplus \mathbb{Z} / 2^{s_{l}}$, and let $n \geq 2$. Consider the Eilenberg-Mac Lane space $K(H, n)$ and its mod 2 cohomology Bockstein spectral sequence $\left\{B_{r}^{*}, d_{r}\right\}$. Suppose that one of the following assumptions holds:

- $n$ is even and $x \in B_{s_{j}}^{n}$ is 0 -transverse for some $1 \leq j \leq l$,

- $x \in P^{e v e n} B_{1}^{*}$ is 0 -transverse $\left(S q^{1} x \neq 0\right)$.

Then $x$ is transverse.

Note that the abelian group $H$ is isomorphic to $\mathbb{Z}^{s} \oplus \mathbb{Z} / 2^{s_{1}} \oplus \cdots \oplus \mathbb{Z} / 2^{s_{l}} \oplus A$, where $A$ is a $2^{\prime}$-torsion group, which is therefore invisible to the mod 2 Bockstein spectral sequence. Hence the first type of 0-transverse elements correspond basically to the fundamental classes $u_{n}$ introduced in Section 2, one for each copy of $\mathbb{Z} / 2^{s_{j}}$ (the fundamental classes coming from the copies of $\mathbb{Z}$ survive to $B_{\infty}^{n}$ ).

Remark 4.3. Theorem 4.2 tells us that very often a 0 -transverse element in the mod 2 cohomology of an Eilenberg-Mac Lane space is transverse and thus detects higher and higher torsion in the integral cohomology. In the cohomology of an arbitrary space though a 0-transverse element is not always transverse. More precisely, the fact that $x \in P^{\text {even }} H^{*}\left(X ; \mathbb{F}_{2}\right)$ is such that $S q^{1} x \neq 0$ does not always force $x$ to be transverse. A counter-example is given by $X=B S O$ and $x=w_{2}$, the second Stiefel-Withney class in $H^{2}\left(B S O ; \mathbb{F}_{2}\right)$. 
From Theorem 4.2 it is not difficult to prove that there is no homology exponent for most Eilenberg-Mac Lane spaces.

Proposition 4.4. Let $H$ be a non-trivial 2-torsion abelian group and let $n \geq 2$. The Eilenberg-Mac Lane space $K(H, n)$ has no homology exponent.

Proof. Accordingly to the Künneth formula, it is sufficient to establish the result when $H=\mathbb{Z} / 2^{s}$ for some $s \geq 1$. If $n$ is even, consider the reduction of the fundamental class $u_{n} \in H^{n}\left(K\left(\mathbb{Z} / 2^{s}, n\right) ; \mathbb{F}_{2}\right)$. This class survives to $B_{s}^{n}$ and is 0 -transverse. Then $u_{n} \in B_{s}^{n}$ is transverse. If $n$ is odd, consider the admissible sequence $(2,1)$. Its excess is exactly 1 and therefore $S q_{s}^{2,1} u_{n} \in P^{\text {even }} H^{*}\left(K\left(\mathbb{Z} / 2^{s}, n\right) ; \mathbb{F}_{2}\right)$ when $n \geq 3$. Moreover we have $S q^{1} S q_{s}^{2,1} u_{n}=S q_{s}^{3,1} u_{n}$ by Adem relations, which means that $S q_{s}^{2,1} u_{n}$ is 0transverse. Hence $S q_{s}^{2,1} u_{n} \in B_{1}^{n+3}$ is transverse.

Proposition 4.5. Let $H$ be a finitely generated abelian group and $n \geq 4$. The Eilenberg-Mac Lane space $K(H, n)$ is then either mod 2 acyclic, or has no homology exponent.

Proof. By the Künneth formula and Proposition 4.4, it is sufficient to analyze the case $H=\mathbb{Z}$. Consider the reduction of the fundamental class $u_{n} \in H^{n}\left(K(\mathbb{Z}, n) ; \mathbb{F}_{2}\right)$. If $n$ is even, then $S q^{2} u_{n}$ is transverse. If $n$ is odd, then $S q^{6,3} u_{n}$ is transverse.

\section{Transverse elements in Postnikov pieces}

We are now ready to prove our main result: Most Postnikov pieces do not have a homology exponent. The strategy to prove this relies on the crucial observation that the transverse implications of certain element in the cohomology of the total space of a fibration can be read in the cohomology of the fibre.

Lemma 5.1. Let $j: F \rightarrow X$ be a continuous map. If $x \in H^{*}\left(X ; \mathbb{F}_{2}\right)$ is such that $j^{*}(x) \neq 0 \in H^{*}\left(F ; \mathbb{F}_{2}\right)$ is transverse, then $x$ itself is transverse.

Proof. It follows from the naturality of the Bockstein spectral sequence.

Theorem 5.2. Let $X$ be an $H$-space of finite type which admits a homology exponent. Then either $X$ is, up to 2-completion, a product of spaces of the form $B \mathbb{Z} / 2^{r}, S^{1}, \mathbb{C} P^{\infty}$ and $K(\mathbb{Z}, 3)$, or $X$ admits infinitely many non-trivial $k$-invariants and homotopy groups. 
Proof. Let us assume that $X$ is a Postnikov piece. By Proposition 1.4, there is an $H$-fibration of the form

$$
Y \longrightarrow X \longrightarrow B \pi_{1} X \times K\left(\mathbb{Z}^{r}, 2\right),
$$

where $Y$ is a simply connected $H$-space of finite type such that $\pi_{2} Y$ is a torsion abelian group. Moreover, $Y$ admits a homology exponent. It is also clearly a Postnikov piece. Let us show that $Y$ is, up to 2-completion, a product of copies of $K(\mathbb{Z}, 3)$. By Proposition 1.2, this will imply that $X$ itself splits as the announced product.

Assume that $\pi_{n} Y=H$ is the highest non-trivial homotopy group of $Y$, up to 2-completion. If $n=2$, since $\pi_{2} Y$ is a torsion abelian group, we deduce from Proposition 4.4 that $H$ is $2^{\prime}$-torsion. In other words $Y_{2}^{\wedge}$ is contractible. We can therefore assume that $n \geq 3$. The space $Y$ fits into the fibration sequence

$$
K(H, n) \stackrel{j}{\longrightarrow} Y \stackrel{i}{\longrightarrow} Y[n-1] \stackrel{k}{\longrightarrow} K(H, n+1),
$$

where $k$ denotes the last $k$-invariant. We analyze the situation in two steps, depending on the presence of 2 -torsion in $H$.

Let us first assume that $H$ contains 2-torsion, let us say $\oplus_{b} \mathbb{Z} / 2^{t_{b}}$. Choose an index $b$ and consider the projection $\pi: H \rightarrow \mathbb{Z} / 2^{t_{b}}$ on the corresponding cyclic subgroup. Pick $v_{n} \in H^{n}\left(K(H, n) ; \mathbb{F}_{2}\right)$, the image via $\pi^{*}$ of the class $u_{n} \in H^{n}\left(K\left(\mathbb{Z} / 2^{t_{b}}, n\right) ; \mathbb{F}_{2}\right)$.

Set $\xi=\left(2^{n-1}-2,2^{n-2}-1,2^{n-3}-1, \ldots, 3,1\right)$. The degree $\operatorname{deg}\left(S q_{t}^{\xi} v_{n}\right)=$ $2^{n}-2$ is even and $S q^{1} S q_{t}^{\xi} v_{n} \neq 0$ since $e(\xi)=n-2$. By Theorem 4.2, the element $S q_{t}^{\xi} v_{n}$ is transverse. Since $Y$ is an $H$-space and the $k$-invariant is an $H$-map, the element $d_{n+1} v_{n}$ is primitive, and so is $d_{2^{n}-1} S q_{t}^{\xi} v_{n}=S q_{t}^{\xi} d_{n+1} v_{n} \in$ $P^{2^{n}-1} H^{*}\left(Y[n-1] ; \mathbb{F}_{2}\right)$. By Proposition $3.5, P^{2^{n}-1} H^{*}\left(Y[n-1] ; \mathbb{F}_{2}\right)=0$ since $2^{n}-1 \in A_{n-1}$. Therefore, $S q_{t}^{\xi} v_{n}$ survives in the Serre spectral sequence and by the previous lemma, $H^{*}\left(Y ; \mathbb{F}_{2}\right)$ contains a transverse element. In particular it has no homology exponent.

Hence, $H$ must be 2-torsion free and is thus isomorphic to $\mathbb{Z}^{s} \oplus A$, where $A$ is a torsion group, for some $s \geq 1$. By Proposition 2.3, $Y$ fits in the following $H$-fibration:

$$
K(H, n) \times K\left(\pi_{n-1} Y, n-1\right) \longrightarrow Y \longrightarrow Y[n-2] .
$$

Choose now $v_{n} \in H^{n}\left(K(H, n) ; \mathbb{F}_{2}\right)$ to be the image of the fundamental class $u_{n} \in H^{n}\left(K(\mathbb{Z}, n) ; \mathbb{F}_{2}\right)$ given by projection on the first copy of $\mathbb{Z}$ in $H$.

If $n \geq 4$, then set

$$
\eta=\left(2^{n-2}+2^{n-3}-2,2^{n-3}+2^{n-4}-1,2^{n-4}+2^{n-5}-1, \ldots, 5,2\right) .
$$


The degree $\operatorname{deg}\left(S q^{\eta} v_{n}\right)=2^{n-1}+2^{n-2}-2$ is even and $S q^{1} S q^{\eta} v_{n} \neq 0$ since $e(\eta)=n-2$. Thus $S q^{\eta} v_{n}$ is transverse and survives in the Serre spectral sequence of the above fibration since $2^{n-1}+2^{n-2}-1 \in A_{n-2}$. In this case, $H^{*}\left(Y ; \mathbb{F}_{2}\right)$ contains a transverse element and has no homology exponent.

Therefore, $n=3$ and $Y \simeq K(H, 3) \times K\left(\pi_{2} Y, 2\right)$. Since $Y$ admits a homology exponent, the torsion group $\pi_{2} Y$ is trivial and $H$ is torsion free.

The proof of the theorem predicts explicit degrees in which to find higher and higher torsion in the integral cohomology of the space.

Corollary 5.3. Let $X$ be a simply connected $H$-Postnikov piece of finite type, say $X \simeq X[n]$. Assume that $\pi_{2} X$ is torsion and that $X$ is not equivalent up to 2-completion to a product of copies of $K(\mathbb{Z}, 3)$. Then, for any integer $k$, there is a copy of $\mathbb{Z} / 2^{k}$ in $H^{*}(X ; \mathbb{Z})$

(1) in degree $2^{k}\left(2^{n}-2\right)$ if $\pi_{n} X$ contains 2 -torsion,

(2) in degree $2^{k}\left(2^{n-1}+2 n-2-2\right)$ if not.

Proof. Since $X$ is different from $K\left(\mathbb{Z}^{m}, 3\right)$, we know from Theorem 5.2 that $X$ has no exponent. The higher and higher torsion is detected by the consecutive powers of the elements $S q^{\xi} v_{n}$ and $S q^{\eta} v_{n}$ constructed in the above proof.

Any finite $H$-space has obviously a homology exponent. Our second corollary applies to its Postnikov sections. As soon as it has at least two homotopy groups, it cannot have a homology exponent.

Corollary 5.4. Let $X$ be a simply connected finite $H$-space and $n \geq 3$. Then $X[n]$ has a homology exponent if and only if $X[n] \simeq X[3] \simeq K\left(\mathbb{Z}^{r}, 3\right)$ for some $r \geq 0$.

Proof. The fact that the $H$-space $X$ is finite and simply connected forces it to be 2 -connected, [3, Theorem 6.10]. Moreover, $\pi_{3} X \cong \mathbb{Z}^{r}$ for some integer $r$, by work of Hubbuck and Kane, [16]. The result now follows directly from Theorem 5.2.

This corollary applies in particular to $S^{3}$. The third Postnikov section $S^{3}[3] \simeq K(\mathbb{Z}, 3)$ has a homology exponent, but all higher Postnikov sections $X[n], n \geq 4$, have none. The following proof of a result obtained by Levi in [20] is, to our knowledge, the first one not based on Miller's solution of the Sullivan's conjecture [22]. Let us mention in this context the work of Klaus, [19], who proves the statement about the $k$-invariants for $B G_{2}^{\wedge}$, not for the loop space. 
Corollary 5.5. Let $G$ be a 2-perfect finite group. Then $\Omega\left(B G_{2}^{\wedge}\right)$ has infinitely many non-trivial $k$-invariants and homotopy groups.

Proof. Suppose $B G_{2}^{\wedge}$ is a Postnikov piece. Following Levi [20], there is a homology exponent for $\Omega\left(B G_{2}^{\wedge}\right)$ and therefore this space has to be a product of copies of $B \mathbb{Z} / 2^{r}, S^{1}, \mathbb{C} P^{\infty}$ or $K(\mathbb{Z}, 3)$. Since $\Omega\left(B G_{2}^{\wedge}\right)$ has torsion homotopy groups, the only copies that can occur are of the form $B \mathbb{Z} / 2^{r}$. Thus $B G_{2}^{\wedge} \simeq K(A, 2)$, where $A$ is a 2-torsion abelian group. By the EvensVenkov theorem, [11], $H^{*}\left(B G_{2}^{\wedge} ; \mathbb{F}_{2}\right)$ is Noetherian. Hence $A$ is trivial, and so is $B G_{2}^{\wedge}$.

\section{Comparison with other forms of Serre's theorem}

In this section we compare our theorem to the other results we mentioned in the introduction. We show that the existence of a homology exponent is stronger than all previously established criteria, except possibly [12], which seems difficult to relate directly to cohomological statements. Therefore, when $X$ is an $H$-space, our result provides new proofs of those. They are very different in spirit, since they do not require the Sullivan conjecture. For simplicity we deal here with simply connected spaces.

Proposition 6.1. Let $X$ be a simply connected $H$-Postnikov piece. Then

(1) (Serre $[27]) H^{*}\left(X ; \mathbb{F}_{2}\right)$ is not finite,

(2) (Lannes-Schwartz [21]) $H^{*}\left(X ; \mathbb{F}_{2}\right)$ is not locally finite,

(3) there exists an element of infinite height in $H^{*}\left(X ; \mathbb{F}_{2}\right)$,

(4) (Grodal [15]) the transcendence degree of $H^{*}\left(X ; \mathbb{F}_{2}\right)$ is infinite unless $X$ is homotopy equivalent, up to 2-completion, to $K(\mathbb{Z}, 2)^{s}$,

(5) (Dwyer-Wilkerson [9]) the unstable module $Q H^{*}\left(X ; \mathbb{F}_{2}\right)$ is not locally finite unless $X$ is homotopy equivalent, up to 2-completion, to $K(\mathbb{Z}, 2)^{s}$.

Proof. Notice first that $K(\mathbb{Z}, 2)$ and $K(\mathbb{Z}, 3)$ satisfy (1)-(5). Assume now that $X$ is a Postnikov piece, say $X \simeq X[n]$. In the proof of Theorem 5.2 we first considered the covering fibration $\left(S^{1}\right)^{r} \rightarrow Y \rightarrow X$. The map $Y \rightarrow X$ induces isomorphisms in homology in high degrees. We can therefore assume that $\pi_{2} X$ is torsion. Our proof then provides a transverse element $x \in H^{*}\left(X ; \mathbb{F}_{2}\right)$ in even degree whose image in $H^{*}\left(K\left(\pi_{n} X, n\right)\right)$ is a transverse element of the form $S q_{t}^{I} u_{n}$ for some admissible sequence $\left(i_{1}, \ldots, i_{m}\right)$. In particular all powers $x^{2^{k}}$ are non-zero, which proves (1), (2), and (3). Moreover the elements $x, S q^{2 i_{1}} x, S q^{4 i_{1}, 2 i_{1}} x, \ldots$ are non-zero, indecomposable, and algebraically independent because so are the corresponding images in $H^{*}\left(K\left(\pi_{n} X, n\right)\right)$. This proves (4) and (5). 


\section{Cohomological finiteness conditions}

The strategy we followed to analyze the integral homology of Postnikov pieces can be applied in a more general context. We work in this last section with simply connected $H$-spaces $X$ such that $H^{*}\left(X ; \mathbb{F}_{2}\right)$ is finitely generated as an algebra over the Steenrod algebra. This section relies on Miller's Theorem [22]. As it may be considered thus as less elementary than the part about Postnikov pieces, we have decided to postpone it till the end of the article.

From the assumption on the mod 2 cohomology, by [5, Lemma 7.1] we infer that there exists an integer $n$ such that the module $Q H^{*}\left(X ; \mathbb{F}_{2}\right)$ of indecomposable elements lies in the $(n-1)$-st stage of the Krull filtration for unstable modules, [25]. Therefore there exists by [5, Theorem 7.3] a simply connected $H$-space $Y=P_{B \mathbb{Z} / 2} X$ with finite mod 2 cohomology and a series of principal $H$-fibrations

$$
X=X_{n} \stackrel{p_{n}}{\longrightarrow} X_{n-1} \rightarrow \cdots \rightarrow X_{1} \stackrel{p_{1}}{\longrightarrow} X_{0}=Y
$$

of simply connected spaces such that the homotopy fiber of $p_{i}$ is an EilenbergMac Lane space $K\left(P_{i}, i\right)$, where $P_{i}$ splits as a product of a finite direct sum $P_{i}^{\prime}$ of cyclic groups $\mathbb{Z} / 2^{r}$ and a finite direct sum $P_{i}^{\prime \prime}$ of Prüfer groups $\mathbb{Z}_{2^{\infty}}$. Let us recall here that $X_{k}$ is obtained as the $\Sigma^{k} B \mathbb{Z} / p$-nullification of $X$ (the above tower is Bousfield's nullification tower, [2]). Since $K\left(\mathbb{Z}_{2^{\infty}}, i\right)$ and $K(\mathbb{Z}, i+1)$ are $\bmod 2$ equivalent, we alter slightly the way in which the $P_{i}$ 's are added to $Y$ in order to work in a more familiar setting. Then we can recover $X$ from the tower

$$
X=Y_{n} \stackrel{q_{n}}{\longrightarrow} Y_{n-1} \rightarrow \cdots \rightarrow Y_{1} \stackrel{q_{1}}{\longrightarrow} Y_{0}=Y
$$

of simply connected spaces and principal $H$-fibrations, where the homotopy fiber of $q_{i}$ is the product of Eilenberg-Mac Lane spaces $K\left(P_{i+1}^{\prime}, i+1\right) \times$ $K\left(P_{i}^{\prime \prime}, i\right)$.

Notice that $Q_{1}=P_{1}^{\prime}$ is trivial because we assume that $X$ is simply connected $\left(Y_{2}^{\wedge}\right.$ is therefore 2-connected, [3]). We have a splitting result, just like in Proposition 2.3.

Lemma 7.1. Let $X$ be a simply connected $H$-space such that $H^{*}\left(X ; \mathbb{F}_{2}\right)$ is finitely generated as an algebra over the Steenrod algebra. Assume that $\pi_{2} X_{2}^{\wedge}$ is torsion. Then there is an $H$-fibration

$$
X \longrightarrow Y_{n-2} \longrightarrow K\left(P_{n+1}^{\prime \prime} \oplus P_{n+1}^{\prime}, n+1\right) \times K\left(P_{n}^{\prime \prime}, n\right) .
$$

Proof. The proof is based on the Zabrodsky lemma, as in Proposition 2.3. 
Our next result is the analog in the present setting of Proposition 3.5. Recall from Section 3 that the set $A_{n}$ consists in those integers for which the 2-adic length is strictly larger than $n$.

Proposition 7.2. Let $X$ be a simply connected $H$-space such that $H^{*}\left(X ; \mathbb{F}_{2}\right)$ is finitely generated as an algebra over the Steenrod algebra. There exists then integers $m$ and $N$ such that $Q^{a} H^{*}\left(X ; \mathbb{F}_{2}\right)=0=P^{a} H^{*}\left(X ; \mathbb{F}_{2}\right)$ for all $a \in A_{n}$ with $a \geq N$.

Proof. The integer $m$ is determined by the stage of the Krull filtration in which $Q H^{*}\left(X ; \mathbb{F}_{2}\right)$ lives, i.e. by the degrees in which the homotopy groups of the homotopy fiber of $X \rightarrow P_{B \mathbb{Z} / 2} X=Y$ are non-trivial. With the above notation, $m=n$ if $P_{n}^{\prime \prime}$ is trivial, and $m=n+1$ if $P_{n}^{\prime \prime}$ is not. The proof goes then by induction on $m$. When $m=0$, choose $N$ to be larger than the cohomological dimension of $Y$. The proof of Proposition 3.5 goes through.

Lemma 7.3. Let $X$ be a simply connected $H$-space which fits, up to 2completion, in an $H$-fibration of the form

$$
K\left(\oplus_{t} \mathbb{Z}, 2\right) \longrightarrow X \longrightarrow Y
$$

where $H^{*}\left(Y ; \mathbb{F}_{2}\right)$ is finite. Then $X$ has no homology exponent unless the fibration splits up to 2-completion, i.e $X \simeq_{2}^{\wedge} Y \times K\left(\oplus_{t} \mathbb{Z}, 2\right)$.

Proof. Let us omit the 2-completions in the proof and write the details of the proof when $t=1$. By the result of Hubbuck and Kane, [16], $\pi_{3} Y$ is isomorphic to a direct sum of say $s$ copies of $\mathbb{Z}$. The map classifying the fibration factors through $Y[3] \simeq K\left(\oplus_{s} \mathbb{Z}, 3\right) \rightarrow K(\mathbb{Z}, 3)$. The $E_{2}$-term of the Serre spectral sequence has the form $\mathbb{Z}[u] \otimes H^{*}(Y ; \mathbb{Z})$, where $u$ has degree 2 and the cohomology of $Y$ is of finite dimension $N$, and of exponent $2^{a}$ for some integer $a$. The differential $d_{3}(u)=x$ for some non-zero element $x \in H^{3}(Y ; \mathbb{Z}) \cong \oplus_{s} \mathbb{Z}$. Therefore $d_{3}\left(u^{n}\right)=n x \otimes u^{n-1}$. At worst $d_{3}\left(x \otimes u^{n-1}\right)$ is non-zero and then hits a torsion element, of order at most $2^{a}$. Hence, on the third column of the $E_{4}$-term, we have a group covering $\mathbb{Z} / 2^{n-a}$ in vertical degree $2^{n}$. From the finiteness of $Y$ we see that the spectral sequence collapses at $E_{N-3}$. An iteration of the above argument shows therefore that the third column of the $E_{\infty}$-term contains a group covering $\mathbb{Z} / 2^{n-(N-5) a}$ in vertical degree $2^{n}$, for any $n \geq 1$. In particular there is arbitrarily high torsion in $H^{*}(X ; \mathbb{Z})$. Therefore, for $X$ to have an exponent, the fibration must split.

Remark 7.4. We point out that the preceding lemma provides simple examples of fibrations, such as $K(\mathbb{Z}, 2) \rightarrow S^{3}\langle 3\rangle \rightarrow S^{3}$, where both the fiber and the base have an exponent, but the total space has none. 
Theorem 7.5. Let $X$ be a simply connected $H$-space of finite type such that $H^{*}\left(X ; \mathbb{F}_{2}\right)$ is finitely generated as an algebra over the Steenrod algebra. Assume that $X$ admits a homology exponent. Then $X$ is, up to 2-completion, the product of a mod 2 finite $H$-space $Y$ with copies of $K(\mathbb{Z}, 2)$ and $K(\mathbb{Z}, 3)$.

Proof. We follow the proof of Theorem 5.2. Let us thus assume that $X$ admits a homology exponent. By killing the copies of $\mathbb{Z}$ in $\pi_{2} X$ just like in Proposition 1.4, we can assume that $\pi_{2} X_{2}^{\wedge}$ is torsion. We also see by inspection of the tower that $\pi_{2}\left(Y_{i}\right)_{2}^{\wedge}$ is torsion for any $i \geq 0$. Therefore the splitting in Lemma 7.1 holds and we work with a fibration

$$
K\left(P_{n}^{\prime \prime} \oplus P_{n}^{\prime}, n\right) \times K\left(P_{n-1}^{\prime \prime}, n-1\right) \longrightarrow X \longrightarrow Y_{n-2} .
$$

If $P_{n}^{\prime} \neq 0$, it must contain a copy of $\mathbb{Z} / 2^{r}$ as direct summand. Choose a power of the corresponding element $S q_{t}^{\xi} v_{n}$, of degree larger than the integer $N$ given in Proposition 7.2. From the Serre spectral sequence for the above fibration we see that this provides a transverse element in $H^{*}\left(X ; \mathbb{F}_{2}\right)$. Therefore $P_{n}^{\prime}=0$ (and so $P_{n}^{\prime \prime}$ is not trivial).

If $n \geq 3$ we choose a copy of $\mathbb{Z}_{2^{\infty}}$ in $P_{n}^{\prime \prime}$ and a suitable power of the corresponding element $S q^{\eta} v_{n}$ to detect a transverse element in $H^{*}\left(X ; \mathbb{F}_{2}\right)$. Since we assume that $X$ has a homology exponent, we see that $n \leq 2$, i.e. $X$ is the homotopy fiber of a map $k: Y \rightarrow K\left(P_{1}^{\prime \prime}, 2\right) \times K\left(P_{2}^{\prime \prime}, 3\right)$. To conclude the proof we must show that this map is trivial.

The mod 2 cohomology of the $H$-space $Y$ is finite. Rationally it is thus a product of odd dimensional spheres and, in particular, $\pi_{4} Y_{2}^{\wedge}$ is torsion. This implies that the projection of $k$ on the second factor $Y \rightarrow K\left(P_{2}^{\prime \prime}, 3\right)$ is the trivial map, up to 2-completion. Hence the copies of $K\left(\mathbb{Z}_{2}^{\wedge}, 3\right)$ split off $X_{2}^{\wedge}$. We are left with the analysis of a fibration $X \longrightarrow Y \longrightarrow K\left(P_{1}^{\prime \prime}, 2\right)$. If the map $Y \rightarrow K\left(P_{1}^{\prime \prime}, 2\right)$ is not trivial, we conclude from Lemma 7.3 that $X$ cannot have a homology exponent. Hence, the fibration must split and this concludes the proof.

\section{References}

[1] Bousfield, A. K.: Nice homology coalgebras. Trans. Amer. Math. Soc. 148 (1970), 473-489.

[2] Bousfield, A. K.: Localization and periodicity in unstable homotopy. J. Amer. Math. Soc. 7 (1994), no. 4, 831-873.

[3] Browder, W.: Torsion in $H$-spaces. Ann. of Math. (2) 74 (1961), 24-51.

[4] Cartan, H.: Algèbres d'Eilenberg-MacLane et homotopie. In Séminaire Henri Cartan de l'Ecole Normale Supérieure (1954-1955), exposés 2-16. Secrétariat mathématique, 11 rue Pierre Curie, Paris, 1955. 
[5] Castellana, N., Crespo, J. A. and Scherer, J.: Deconstructing Hopf spaces. Invent. Math. 167 (2007), no. 1, 1-18.

[6] Castellana, N., Crespo, J. A. and Scherer, J.: On the cohomology of highly connected covers of finite Hopf spaces. Adv. Math. 215 (2007), no. 1, 250-262.

[7] Clément, A.: Integral cohomology of finite Postnikov towers. Ph. D. Thesis, University of Lausanne, Switzerland, 2002.

[8] Clément, A.: Integral cohomology of 2-local Hopf spaces with at most two non-trivial finite homotopy groups. In An alpine anthology of homotopy theory, 87-99. Contemp. Math. 399. Amer. Math. Soc., Providence, RI, 2006.

[9] Dwyer, W. G. and Wilkerson, C. W.: Spaces of null homotopic maps. Astérisque 191 (1990), no. 6, 97-108. International Conference on Homotopy Theory (Marseille-Luminy, 1988).

[10] Dwyer, W. G.: The centralizer decomposition of BG. In Algebraic topology: new trends in localization and periodicity (Sant Feliu de Guíxols, 1994), 167-184. Progr. Math. 136. Birkhäuser, Basel, 1996.

[11] Evens, L.: The cohomology ring of a finite group. Trans. Amer. Math. Soc. 101 (1961), 224-239.

[12] Félix, Y., Halperin, S., Lemaire, J. M. and Thomas, J. C.: Mod $p$ loop space homology. Invent. Math. 95 (1989), no. 2, 247-262.

[13] Félix, Y., Halperin, S. and Thomas, J. C.: Torsion in loop space homology. J. Reine Angew. Math. 432 (1992), 77-92.

[14] Ganea, T.: A generalization of the homology and homotopy suspension. Comment. Math. Helv. 39 (1965), 295-322.

[15] Grodal, J.: The transcendence degree of the mod $p$ cohomology of finite Postnikov systems. In Stable and unstable homotopy (Toronto, ON, 1996), 111-130. Fields Inst. Commun. 19. Amer. Math. Soc., Providence, RI, 1998.

[16] Hubbuck, J. R. and Kane, R.: On $\pi_{3}$ of a finite $H$-space. Trans. Amer. Math. Soc. 213 (1975), 99-105.

[17] Kane, R.: The homology of Hopf spaces. North-Holland Mathematical Library 40. North-Holland Publishing Co., Amsterdam, 1988.

[18] Kane, R.: Personal communication. November 2006.

[19] Klaus, S.: A generalization of the non-triviality theorem of Serre. Proc. Amer. Math. Soc. 130 (2002), no. 5, 1249-1256 (electronic).

[20] Levi, R.: On finite groups and homotopy theory. Mem. Amer. Math. Soc. 118 (1995), no. 567.

[21] Lannes, J. And Schwartz, L.: À propos de conjectures de Serre et Sullivan. Invent. Math. 83 (1986), no. 3, 593-603.

[22] Miller, H.: The Sullivan conjecture on maps from classifying spaces. Ann. of Math. (2) 120 (1984), no. 1, 39-87. 
[23] Milnor, J. W. And Moore, J. C.: On the structure of Hopf algebras. Ann. of Math. (2) 81 (1965), 211-264.

[24] McGibbon, C. A. And Neisendorfer, J. A.: On the homotopy groups of a finite-dimensional space. Comment. Math. Helv. 59 (1984), no. 2, 253257.

[25] Schwartz, L.: Unstable modules over the Steenrod algebra and Sullivan's fixed point set conjecture. Chicago Lectures in Mathematics. University of Chicago Press, Chicago, IL, 1994.

[26] SElick, P.: Moore conjectures. In Algebraic topology-rational homotopy (Louvain-la-Neuve, 1986), 219-227. Lecture Notes in Math. 1318. Springer, Berlin, 1988.

[27] Serre, J. P.: Cohomologie modulo 2 des complexes d'Eilenberg-MacLane. Comment. Math. Helv. 27 (1953), 198-232.

[28] Smith, L.: Lectures on the Eilenberg-Moore spectral sequence. Lecture Notes in Mathematics 134. Springer-Verlag, Berlin-New York, 1970.

[29] Spanier, E. H.: Algebraic topology. Springer-Verlag, New York-Berlin, 1981. Corrected reprint.

Recibido: 15 de junio de 2007

Revisado: 4 de septiembre de 2007

\author{
Alain Clément \\ Louis-Meyer 9 \\ CH-1800 Vevey, Switzerland \\ alain.clement@bluewin.ch \\ Jérôme Scherer \\ Departament de Matemàtiques \\ Universitat Autònoma de Barcelona \\ E-08193 Bellaterra, Spain \\ jscherer@mat.uab.es
}

The second author is supported by the program Ramón y Cajal, MEC, Spain, and FEDER/MEC grant MTM2007-61545. This research was partially supported by the Swiss National Science Foundation grant FN 200020-105383. 